Results: Our results demonstrate that early exposure to S100A8 interferes with in-vitro differentiation of moDCs. Compared to controls S100A8-exposed moDCs show dramatically reduced surface expression of co-stimulatory molecules upon LPS-induced maturation. In addition, early treatment of moDCs with S100A8 alters the secretion of immune-regulatory cytokines and chemokines depending on the developmental state of moDCs. S100A8-induced effects on moDC maturation are not limited to TLR4 stimulation but rather trigger a common state of unresponsiveness. Furthermore, mitochondrial respiration and glycolytic function is diminished in S100A8-treated moDCs.

As a consequence, S100A8-exposed moDCs have a reduced potential to induce autologous T-cell proliferation. We can show that these differences are mainly caused by reduced surface expression of co-stimulatory molecules on S100A8-treated moDCs.

Mechanistically, genome-wide gene expression analysis reveals dramatic differences in gene expression between S100A8-exposed and conventionally differentiated moDCs. We demonstrate that $\mathrm{S} 100 \mathrm{~A} 8$ pre-treatment of moDCs significantly blocks LPS-induced gene expression during moDC activation. Interestingly, insilico analysis of transcription factor networks predicts $N F_{\kappa} B$ and $\mathrm{C} / \mathrm{EBP} \delta$ as master regulators of S100A8-induced effects in developing moDCs. C/EBP $\delta$ on protein level, indeed, shows reduced expression in S100A8-differentiated moDCs prior and after LPS-induced maturation when compared to conventionally differentiated moDCs.

Conclusions: Taken together, our results demonstrate a novel regulatory mechanism of innate immunity to prevent overwhelming immune responses. Dysregulated repression of detrimental adaptive immune responses might very well contribute to the disease phenotype in auto-immune disorders with high systemic S100A8/A9 levels. Therefore, S100A8-differentiated immune-suppressive DCs potentially represent a promising therapeutic tool to treat auto-immune diseases in the future.

Disclosure of Interest: None declared

DOI: 10.1136/annrheumdis-2017-eular.4960

\section{OP0092 INTERLEUKIN 37 REVERSES THE METABOLIC COST OF INFLAMMATION, INCREASES OXIDATIVE RESPIRATION AND IMPROVES EXERCISE TOLERANCE}

G. Cavalli ${ }^{1}$, D. Ballak ${ }^{2}$, J.J. Justice ${ }^{2}$, K. Boyle ${ }^{3}$, A. D'Alessandro ${ }^{3}$, L.A. Joosten ${ }^{4}$, L. Dagna ${ }^{1}$, C.A. Dinarello ${ }^{5}{ }^{1}$ Unit of Immunology, Rheumatology, Allergy and Rare Diseases (UniRAR), Vita-Salute San Raffaele University, Milan, Italy; ${ }^{2}$ University of Colorado Boulder, Boulder, $\mathrm{CO} ;{ }^{3}$ University of Colorado Denver, Aurora, CO, United States; ${ }^{4}$ Radboud University Medical Center, Nijmegen, Netherlands; ${ }^{5}$ Medicine, University of Colorado Denver, Aurora, CO, United States

Background: The IL-1 family cytokine interleukin 37 (IL-37) has broad antiinflammatory effects and functions as a natural suppressor of innate inflammation and acquired immunity (1). We have reported that administration of recombinant human IL-37 to wild type mice or expression in mice transgenic for human IL-37 suppress proinflammatory cytokines and curb excessive inflammation in various conditions including inflammatory arthritis (2). Besides these anti-inflammatory effects, IL-37 also induces complex effects on metabolism. In particular, IL-37 can directly activate AMP-activated protein kinase (AMPK), a central regulator of cellular energy homeostasis and exercise-regulated metabolism (3).

Objectives: In this study, we evaluate the effects of IL-37 treatment on exercise tolerance in mice with systemic inflammation induced by LPS injection. We further investigate the effects of IL-37 on exercise tolerance in healthy mice, with specific focus on the metabolic changes induced by IL-37 administration and possibly responsible for a reduction in the metabolic costs of inflammation.

Results: Exogenous administration of IL-37 to healthy mice, not subjected to an inflammatory challenge, also improved exercise performance by $82 \%$ compared to vehicle-treated mice $(p=0.01)$. Treatment with 8 daily doses of IL-37 resulted in a further $326 \%$ increase in endurance running time compared to the performance level of mice receiving vehicle $(p=0.001)$. These properties required the engagement of the IL-1 decoy receptor 8 (IL-1R8) and the activation of AMP-activated protein kinase (AMPK), since both inhibition of AMPK and IL-1R8 deficiency abrogated the positive effects of IL-37 on exercise performance. Mechanistically, treatment with IL-37 induced marked metabolic changes with higher levels of muscle AMPK, greater rates of oxygen consumption and increased oxidative phosphorylation. Metabolomic analyses of plasma and muscles of mice treated with IL-37 revealed an increase in AMP/ATP ratio, reduced levels of pro-inflammatory mediator succinate and oxidative stress-related metabolites as well as changes in amino acid and purine metabolism.

Conclusions: These effects of IL-37 to limit the metabolic costs of chronic inflammation and to foster exercise tolerance provide a rationale for therapeutic use of IL-37 in the treatment of inflammation-mediated fatigue.

References:

[1] Dinarello CA, et al. (2016) Suppression of innate inflammation and immunity by interleukin-37. Eur J Immunol.

[2] Cavalli G, et al. (2016) Treating experimental arthritis with the innate immune inhibitor interleukin-37 reduces joint and systemic inflammation. Rheumatology (Oxford).

[3] Ballak DB, et al. (2014) IL-37 protects against obesity-induced inflammation and insulin resistance. Nature communications.
Disclosure of Interest: None declared

DOI: 10.1136/annrheumdis-2017-eular.1789

\section{OP0093 IL-38 OVEREXPRESSION INDUCES ANTI-INFLAMMATORY EFFECTS IN MICE ARTHRITIS MODELS AND IN HUMAN MACROPHAGES IN VITRO}

M.-A. Boutet ${ }^{1}$, A. Najm ${ }^{2}$, G. Bart ${ }^{2}$, R. Brion ${ }^{1}$, S. Touchais $^{2}$, V. Trichet ${ }^{1}$, P. Layrolle ${ }^{1}$, C. Gabay ${ }^{3}$, G. Palmer-Lourenco ${ }^{3}$, F. Blanchard ${ }^{1}$, B. Le Goff ${ }^{2}$. ${ }^{1}$ Inserm UMR957, Université de Nantes; ${ }^{2}$ Rheumatology Unit, Nantes University Hospital, Nantes, France; ${ }^{3}$ Department of pathology and immunology, University of Geneva school of medicine, Geneva, Switzerland

Background: IL-38 is a newly characterized cytokine that belongs to the IL-1 family. This cytokine is expressed in the rheumatoid arthritis (RA) synovial tissue (1) and IL-38 deficient mice have exacerbated arthritis (2).

Objectives: This work aims to analyze the effect of IL-38 overexpression in the joints of arthritic mice, in human macrophages and synovial fibroblasts in vitro. Methods: Articular injections of an adeno-associated virus (AAV2/8) encoding IL-38 were performed in Collagen-Induced Arthritis (CIA), K/BxN Serum TransferInduced Arthritis (STIA) and Antigen-Induced Arthritis (AIA) in mice. The effect of IL-38 overexpression was evaluated through clinical scores, immunohistochemistry, microCT, Luminex and RT-qPCR analysis. THP-1 monocytes/macrophages were transduced with a lentiviral vector to overexpress IL-38. Effect of conditioned media from these transduced THP-1 cells was also tested on primary culture: M1 macrophages and fibroblast-like synoviocytes from RA patients (RA-FLS).

Results: Clinical inflammatory scores were significantly decreased after AAV IL-38 injection in joints of mice with CIA and STIA, but not AIA. This decrease was accompanied by reduced macrophage infiltration and a decreased expression of Th17 expressed cytokines (IL-17, IL-23, IL-22, TNF $\alpha$ ). However, IL-38 overexpression had no effect on cartilage or bone destruction. In vitro, the THP-1 monocytic cell line expressed less IL-6, TNF $\alpha$ and IL-23 after IL-38 overexpression. Conditioned media from these cells, containing released IL-38, was also able to exert an anti-inflammatory effect on human primary M1 macrophages and RA-FLS by reducing their IL-23 (M1) and IL-6 (M1 and RA-FLS) expression.

Conclusions: This study shows for the first time that IL-38 overexpression attenuates the severity of experimental arthritis in two mice arthritis models. IL-38 may exert its anti-inflammatory effects by decreasing the production of pro-inflammatory cytokines by macrophages and synovial fibroblasts. This effect can lead to the development of novel treatment strategies in arthritis.

\section{References:}

[1] Boutet MA, Bart G, Gahier M, Amiaud J, Brulin B, Charrier C, Morel F, Lecron JC, RolliDerkinderen M, Boureille A, et al. Distinct expression of IL-36 $\alpha, \beta$, $\gamma$ and their antagonists IL-36Ra and IL-38 in Psoriasis, Rheumatoid Arthritis and Crohn's disease. Clin Exp Immunol, 2015. doi: 10.1111/cei.12761.

[2] Takenaka, S., Kaieda, S., Kawayama, T., Matsuoka, M., Kaku, Y., Kinoshita, T., Sakazaki, Y., Okamoto, M., Tominaga, M., Kanesaki, K., et al. (2015). IL-38: A new factor in rheumatoid arthritis. Biochem. Biophys. Rep. 4, 386-391.

Disclosure of Interest: None declared

DOI: 10.1136/annrheumdis-2017-eular.2257

\section{OP0094 DEVELOPMENT OF A 37-CHANNEL MASS CYTOMETRY (CYTOF) PANEL TO PREDICT TREATMENT RESPONSE IN RHEUMATOID ARTHRITIS}

B. Mulhearn ${ }^{1,2,3}$, S. Viatte ${ }^{3}$, T. Hussell ${ }^{1}$, A. Barton ${ }^{2,3},{ }^{1}$ Manchester Collaborative Centre for Inflammation Research (MCCIR), University of Manchester; ${ }^{2}$ Kellgren Centre for Rheumatology, Central Manchester University Hospitals NHS Foundation Trust; ${ }^{3}$ Centre for Musculoskeletal Research, Division of Musculoskeletal \& Dermatological Sciences, University of Manchester, Manchester, United Kingdom

Background: It is well-established that reducing the inflammation of rheumatoid arthritis (RA) early leads to improved patient outcomes. Patients who fail to respond to conventional DMARDs move on to biologic drugs, however $30 \%$ will fail to respond to the first drug tried. Biologics are trialled for at least 3 months before their efficacy can be assessed, during which time irreversible joint damage may occur.

Currently there is no way to predict which drug will be effective in individual patients. It is therefore a research priority to develop a method to predict response to each class of biologic drug. I hypothesise that the immune phenotype of a patient will influence treatment response.

Objectives: This study aims to immunophenotype T cells using mass cytometry and to test novel unbiased methods of analysing high-dimensional data.

Methods: Ten healthy controls $(\mathrm{HC})$ and $10 \mathrm{RA}$ patients with established disease were included. T cells were isolated and stimulated for 4 hours using anti-CD3/anti-CD28 beads in the presence of monensin and brefeldin A. Cells were stained with a 37-channel mass cytometry panel including surface markers, intracellular antigens and transcription factors

Analysis was performed by conventional biaxial gating alongside tools available on the MRC Cytobank platform, namely Visual t-distributed stochastic neighbour embedding (viSNE), and Cluster identification, characterization, and regression (CITRUS). The CITRUS analysis compared stimulated HC with RA T cells. To 
compare abundances of cell clusters, the association model Prediction Analysis for Microarrays (PAMR) was applied with a minimum cluster size of $2.2 \%, 5$ cross-validation folds and a false discovery rate of $1 \%$.

Results: Conventional biaxial gating showed that there were large differences in the proportions of both IFN $\gamma^{+}$CD4 T cells (1-15\%) and IL-17A $A^{+}$CD4 T cells $(0-9 \%)$ within and between RA and HC. The proportion of IFN $\gamma^{+}$CD4 T cells (Th1) did not correlate with that of IL-17A + T cells (Th17), leading to the generation of 4 immunophenotypes: "Th1", "Th17", "double-hi", and "low", determined by cytokine expression.

Using CITRUS we identified 3 clusters of cells which differed significantly in abundance between $\mathrm{HC}$ and RA. Cluster 1 was $\mathrm{CD} 4^{+} \mathrm{CD} 38^{+}$, had characteristics of regulatory $\mathrm{T}$ cells and was more abundant in $\mathrm{HC}$. Cluster 2 was $\mathrm{CD} 28^{\text {low }} \mathrm{CD}^{+}$ expressing perforin and Tbet, and cluster 3 a $\mathrm{CD} 127^{\text {high }} \mathrm{CCR} 6^{+}$population, both of which were more abundant in RA.

Conclusions: Cytokine expression of ex vivo stimulated T cells from RA and HC is highly variable and can be detected using mass cytometry. Cytokine signatures of this kind may be informative when predicting treatment response.

CITRUS identified 3 cell clusters which may have been missed using conventional methods of analysis. Importantly, CITRUS allowed inspection of the phenotype of each cluster.

Our next step is to compare RA responders to non-responders using the methods described. The function of these clusters will be further investigated by cell isolation with fluorescence-activated cell sorting (FACS) and may go some way in predicting treatment response. Finally we recommend the use of both automated clustering algorithms alongside conventional gating methods when analysing high-dimensional data.

Disclosure of Interest: None declared

DOI: 10.1136/annrheumdis-2017-eular.2067

\section{OP0095 PREDICTION OF CONNECTIVE TISSUE DISEASE IN AN AT-RISK COHORT USING A NOVEL INTERFERON STIMULATED GENE EXPRESSION SCORE}

M.Y. Md Yusof, Y. El-Sherbiny, A. Psarras, E. Hensor, A. Alase, A. Mohamed, M. Wittmann, P. Emery, E.M. Vital. Leeds Institute of Rheumatic and Musculoskeletal Medicine, University of Leeds, Leeds, United Kingdom

Background: A period of ANA positivity and other immune dysregulation precedes connective tissue disease, providing a potential opportunity for disease prevention. Type I interferons (IFN-I) play a role in pathogenesis but their role in disease initiation is unclear.

Objectives: To develop biomarkers of progression to systemic autoimmunity, with a view to enabling early intervention for disease prevention.

Methods: A prospective observational study was conducted in 125 patients At Risk of CTD defined by (i) ANA; (ii) $\leq 1$ clinical SLE criteria; (iii) symptom duration $<12$ months and (iv) treatment-naïve. Progression was defined by meeting 2012 ACR/SLICC SLE, 2016 ACR/EULAR Primary Sjogren's, or other diagnostic criteria. Expression of 30 selected="selected" ISGs was measured using Taqman. Factor analysis was used to reduce the gene expression data to a limited set of factors, which were compared between patient groups using Mann Whitney $U$ Test. Two factor scores explained $80 \%$ of the data variance; "Score A" (composed of IFN- $\alpha$ responsive genes) and "Score B" (genes responsive to IFN- $\alpha$ and $\gamma$ ). 95 healthy controls and 107 SLE patients were used as negative and positive controls. Results: 82 patients with 1-year follow-up data were studied. 71 were female with mean age $48 \pm 15$ years. $16(20 \%)$ patients progressed to CTD (SLE=12, Sjogren's=4) in the following 12 months. At baseline, only IFN Score A was increased in both At Risk-CTD and SLE vs healthy controls; $p<0.001$. IFN Score $B$ was only increased in true established SLE.

In At-Risk patients, IFN Score B was low in patients who did not progress and increased in those who did progress; $p=0.004$. However, there was no difference in IFN Score A between these two groups; $p=0.252$ (Figure 1). Although complement levels and lymphocyte counts were lower in SLE, these were not different between the At-Risk progression and non-progression groups. Anti-dsDNA titres were higher in SLE but not different between the progression groups; all $p>0.10$.
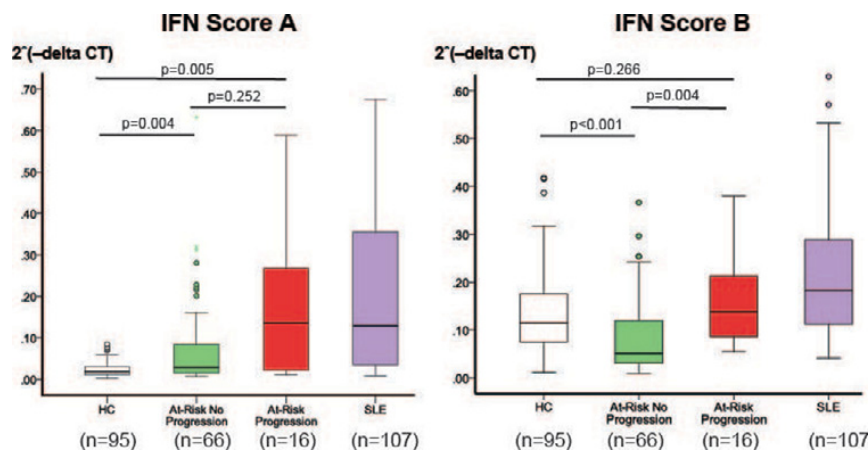

Conclusions: A novel ISG score predicts progression from ANA+ to clinical autoimmune disease. This may allow early intervention to prevent CTD. Analysis of other clinical, immunological and imaging biomarkers are in progress as well as a validation cohort.

Disclosure of Interest: M. Y. Md Yusof: None declared, Y. El-Sherbiny Grant/research support from: AstraZeneca, A. Psarras: None declared, E. Hensor: None declared, A. Alase: None declared, A. Mohamed: None declared, M. Wittmann: None declared, P. Emery Grant/research support from: AstraZeneca, E. Vital Grant/research support from: AstraZeneca DOI: 10.1136/annrheumdis-2017-eular.6925

\section{OP0096 INTERFERON SIGNATURE IN SYSTEMIC SCLEROSIS LUNG MICROVASCULAR ENDOTHELIAL CELLS}

F.A. Mendoza ${ }^{1}$, S. Piera-Velazquez ${ }^{2}$, P. Wermuth ${ }^{2}$, S. Addya ${ }^{3}$,

C. Feghali-Bostwick ${ }^{4}$, S.A. Jimenez ${ }^{2}$. 'Division of Rheumatology and

Scleroderma Center; ${ }^{2}$ Jefferson Institute of Molecular Medicine and Scleroderma Center; ${ }^{3}$ Kimmel Cancer Center, Thomas Jefferson University, Philadelphia;

${ }^{4}$ Division of Rheumatology \& Immunology, Medical University of South Carolina, Charleston, United States

Background: Systemic Sclerosis (SSc) is characterized by severe fibroproliferative vasculopathy, exaggerated deposition of extracellular matrix molecules (ECM) in skin and multiple internal organs, and alterations of humoral, cellular and innate immunity. Vascular changes are responsible for the earliest and most severe SSc clinical manifestations, however, the mechanisms responsible have not been elucidated.

Objectives: The goal of this study was to analyze the gene expression differences between normal and SSc lung microvascular endothelial cells (EC) to improve the understanding of SSc vasculopathy pathophysiology.

Methods: Pulmonary microvascular EC were isolated employing immunomagnetic procedures from lungs from patients with SSc undergoing lung transplantation. Control pulmonary microvascular EC were isolated from autopsies of individuals who died from non-pulmonary causes. Following isolation, microarrays were performed in EC from each group. Expression of genes with the highest differential expression was validated with RT-PCR and Western blots.

Results: Interferon-stimulated genes (ISGs) including IFI44L, IFI44, IFI6, IFIH1, IFIT1 displayed the highest differential expression; being overexpressed in EC obtained from the three SSc donors (Figure 1). Other genes such as those encoding ECM production related proteins, genes associated with posttranslational methylation, and genes for numerous chemokines and cytokines were also differentially overexpressed in SSc EC. Increased gene expression and increased protein levels of selected ISGs were confirmed by Western blots.

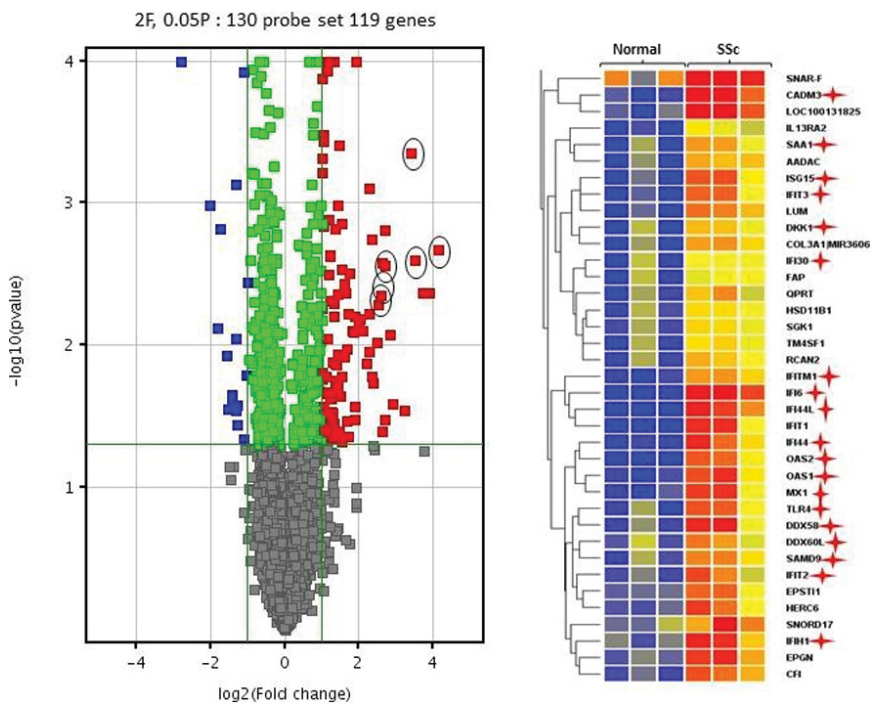

Figure 1. A: Volcano Plot showing differentially expressed transcripts with 2 fold or greater difference $(p<0.05)$ in expression between SSc and normal control microvascular ECs (130 transcripts corresponding to119 genes). Selected ISGs are encircled (IFI-6, IFIT1, IFI-44,IFI44L, IFIT3, OAS-1) B: Detail of the resulting heat map of a dendrogram (hierarchically clustered) reveals groups of genes with high expression levels (red squares), low expression levels (blue squares) or background expression levels (yellow squares). Interferon related genes are marked with a red star.

Conclusions: Numerous ISGs are differentially overexpressed in SSc pulmonary microvascular EC in comparison with normal control EC. These results suggest that events leading to an interferon response in these cells may play a role in the pathogenesis of SSc lung vasculopathy.

References:

[1] Kahaleh B. Vascular Disease in Scleroderma: Mechanisms of Vascular Injury. Rheumatic Disease Clinics of North America 2008; 34(1):57-71.

[2] Trojanowska M. Cellular and molecular aspects of vascular dysfunction in systemic sclerosis. Nat Rev Rheumatol 2010; 6(8):453-60. 